\title{
Densidad y estructura de las poblaciones de tortuga pímpano (Chelydra acutirostris Peters 1862) (Chelydridae) en las quebradas Cajones y Los Coclí, departamento del Quindío, Colombia
}

\author{
Density and structure of the pimpano's turtle populations \\ (Chelydra acutirostris Peters 1862) (Chelydridae) in the Cajones streams \\ and The Coclí, department of Quindío, Colombia
}

\author{
Katherine Young-Valencia ${ }^{1}$, Andrés Fernando Ortega ${ }^{2}$, Álvaro Botero-Botero ${ }^{3,4}$ \\ Resumen
}

\begin{abstract}
Objetivo: Se estudió la densidad y estructura de la población de tortugas pímpano capturadas y avistadas en las quebradas Cajones (municipio de Montenegro) y Los Coclí (municipio de Quimbaya), departamento del Quindío. Metodología: La fase de campo se llevó a cabo desde abril hasta septiembre del año 2012, donde se realizaron 36 salidas al campo, con duración de una hora aproximadamente por cada 400 metros cuadrados por trayecto muestreado. La quebrada Los Coclí tiene una longitud de casi 2000 metros y en estos se establecieron dos trayectos. A su vez, en la quebrada Cajones, con longitud aproximada de 3000 metros, se establecieron cuatro trayectos; cada trayecto tenía 400 metros de longitud con un ancho promedio del cauce medido cada $10 \mathrm{~m}$. Los trayectos fueron separados por un espacio de 200 metros. Todas las tortugas se capturaron manualmente. Cada tortuga fue medida y marcada con corte rectangular en el caparazón. Se estimó la densidad mediante la relación entre el número de tortugas capturadas y avistadas por el área. Resultados: La densidad para la quebrada Cajones fue 423,77 individuos $/ \mathrm{km}^{2}$. Para la quebrada Los Coclí la densidad fue $275,34 \mathrm{ind} / \mathrm{km}^{2}$. Se capturados en la quebrada Cajones 15 adultos y cinco juveniles; se encontró una alta relación entre las variables longitud del caparazón y peso en individuos de menor tamaño. Las hembras presentaron mayor longitud del caparazón comparado con los machos, pero la longitud del plastrón y la longitud preanal aumentó una proporción más rápida en machos. Para la quebrada Los Coclí solo se logró capturar tres juveniles.
\end{abstract}

Palabras clave: Chelydra acutirostris, Densidad tortugas, Estructura tortugas, Hábitat de tortugas, Quebradas.

\begin{abstract}
Objective: The habitat, popultion density, and structure of the Pimpano's turtle where studied at the Cajones (in Montenegro) and Los Coclí (in Quimbaya) streams in the department of Quindío. Methodology: Field work was conducted from April to September of 2012 for a total of 36 field trips, lasting one hour each 400 square meters per transect sampled. A total of six sampling transects of $400 \mathrm{~m}$ were established along the course of the streams, two transects along the $2000 \mathrm{~m}$ of Los Coclí stream, and four transects in the stream the Cajones with an approximate length of $3000 \mathrm{~m}$. The transects were separated $200 \mathrm{~m}$. All turtles were caught manually. Each turtle was measured and marked with a rectangular cut in the shell. Density was estimated by the relation between the number of turtles caught and sighted in each transect. Results: The density for the Cajones stream was 423,77 individuals $/ \mathrm{km}^{2}$, and for Los Coclí stream the density was 275,34 individuals $/ \mathrm{km}^{2}$. At the Cajones stream, 15 adults and five juveniles were captured. We found a high relationship between carapace length and weight in smaller individuals. We also detected that female individuals had larger carapace length compared to males, but the length of the plastron and preanal length increases

\footnotetext{
Programa de Biología, Universidad del Quindío, Colombia.e-mail: katherineyoungv@ hotmail.com

Fundación ARCHELON, Cali, Colombia. e-mail: afog71@ @otmail.com

Grupo de investigación Biodiversidad y Educación Ambiental (BIOEDUQ), Universidad del Quindío; Fundación Neotrópica, La Tebaida, Colombia. e-mail: abotero@uniquindio.edu.co

Fecha recepción: Septiembre 17, 2013

Fecha de aprobación: Diciembre 12, 2013 Editor asociado: Jiménez AM
} 
more rapidly in males. For the stream Los Coclí we only report the capture of three juvenile turtles.

Keywords: Chelydra acutirostris, Turtle's density, Turtle's structure, Streams.

\section{Introducción}

La tortuga acuática Chelydra acutirostris es una de las tortugas más grandes presentes en la zona andina, con un gran tamaño de cabeza, miembros y maxilar fuerte y ganchudo para agarrar a sus presas. Se caracteriza por su cola larga y caparazón bien desarrollado y su plastrón reducido y cruciforme (Rueda-Almonacid et al. 2007). Se encuentra distribuida en Honduras, Nicaragua, Costa Rica, Panamá Colombia y Ecuador (Medem 1977).

En Colombia, esta tortuga ocasionalmente es cazada como fuente de alimento por las comunidades del Pacífico y el Caribe colombianos y ha sido sobreexplotada en la zona del río Cauca (RuedaAlmonacid et al. 2007). Además, en las madres viejas, áreas pantanosas las cuales son uno de los hábitats preferidos de estos reptiles, sus poblaciones han disminuido por la agricultura mecanizada, ganadería extensiva, infraestructura de riego y los cultivos extensivos de caña de azúcar y la contaminación de los cuerpos de agua (Henao y Ruiz 1996, RuedaAlmonacid et al. 2007). En la actualidad la tortuga pímpano está siendo presionada en los departamentos del Quindío y Valle del Cauca, por el incremento de la población humana y las precarias condiciones económicas de los campesinos lo que la convierte en una fuente alternativa de proteína de origen animal (Castaño-M 2002).

Con estos antecedentes se pretendió evaluar la densidad y estructura de las poblaciones de tortuga pímpano en dos quebradas del departamento del Quindío, Colombia.

\section{Metodología}

Área de estudio. El área de estudio se circunscribe en la cuenca hidrográfica del río La Vieja, alto Cauca, Colombia, donde fueron seleccionadas dos quebradas en las que se tiene registro de la presencia de la especie; la primera fue la quebrada Cajones (N 04 32’22.08", W 7546’6.28, entre 997-1250 metros de elevación) que aflora después del casco ur- bano del municipio de Montenegro (departamento del Quindío) y en la zona media recibe las aguas residuales del municipio de Montenegro a través de la quebrada Chochalito y tras un recorrido de $3000 \mathrm{~m}$ desemboca en el río Espejo en los predios aledaños del Parque Nacional de la Cultura Cafetera. El segundo afluente fue la quebrada Los Coclí un tributario del río Roble que se encuentra ubicada dentro de los predios de la hacienda El Ocaso, vereda el Laurel, municipio de Quimbaya (N 4³4'08', W 7551'03') a 970 metros de elevación. Esta es una quebrada de tipo primario que cuenta con una longitud aproximada de 2000 m (Figura 1).

Muestreo. Entre abril y septiembre del año 2012, se llevaron a cabo tres muestreos mensuales (dos diurnos y uno nocturno) en cuatro trayectos en la quebrada Cajones y dos en la quebrada Los Coclí para un total de 18 muestreos en cada quebrada. El esfuerzo de muestreo fue evaluado como horas/hombre, con intensidad aproximadamente de una hora para cada $400 \mathrm{~m}$.

Se establecieron trayectos de $400 \mathrm{~m}$ de longitud con el ancho promedio del canal, los cuales fueron separados entre sí por $200 \mathrm{~m}$ de longitud para generar independencia de los datos. El número de estos fue determinado por la longitud total de cada quebrada desde donde aflora hasta donde desemboca, adecuados de acuerdo a la orientación y disposición de la topografía del terreno. En cada unidad de muestreo realizaron caminatas por el borde del curso del agua en contra de la corriente, para asegurar el avistamiento y captura de tortugas diferentes, buscando aquellos sitios preferidos por la especie (palizadas, zonas pantanosas, arenosas, con pocas piedras, con poca corriente y no muy profunda).

Los sitios de captura y avistamiento de cada tortuga fueron georeferenciados con la ayuda de un GPS (Garmin Etrex High Sensitivity), los registros se transfirieron a cartografía digital de la zona y se diseñó un mapa con la distribución de la especie en el programa ARCVIEW GIS 3.2 (Bandas 2003, Antúnez y Mendoza 1992).

La captura se realizó con una vara y una red manual de acuerdo con las recomendaciones de Rueda-Almonacid et al. (2007). Para la determinación sexual, se midió la longitud preanal y la longitud del plastrón en individuos adultos reproductivos (longitud del caparazón, $L C>20 \mathrm{~cm}$ ), porque la longitud preanal 


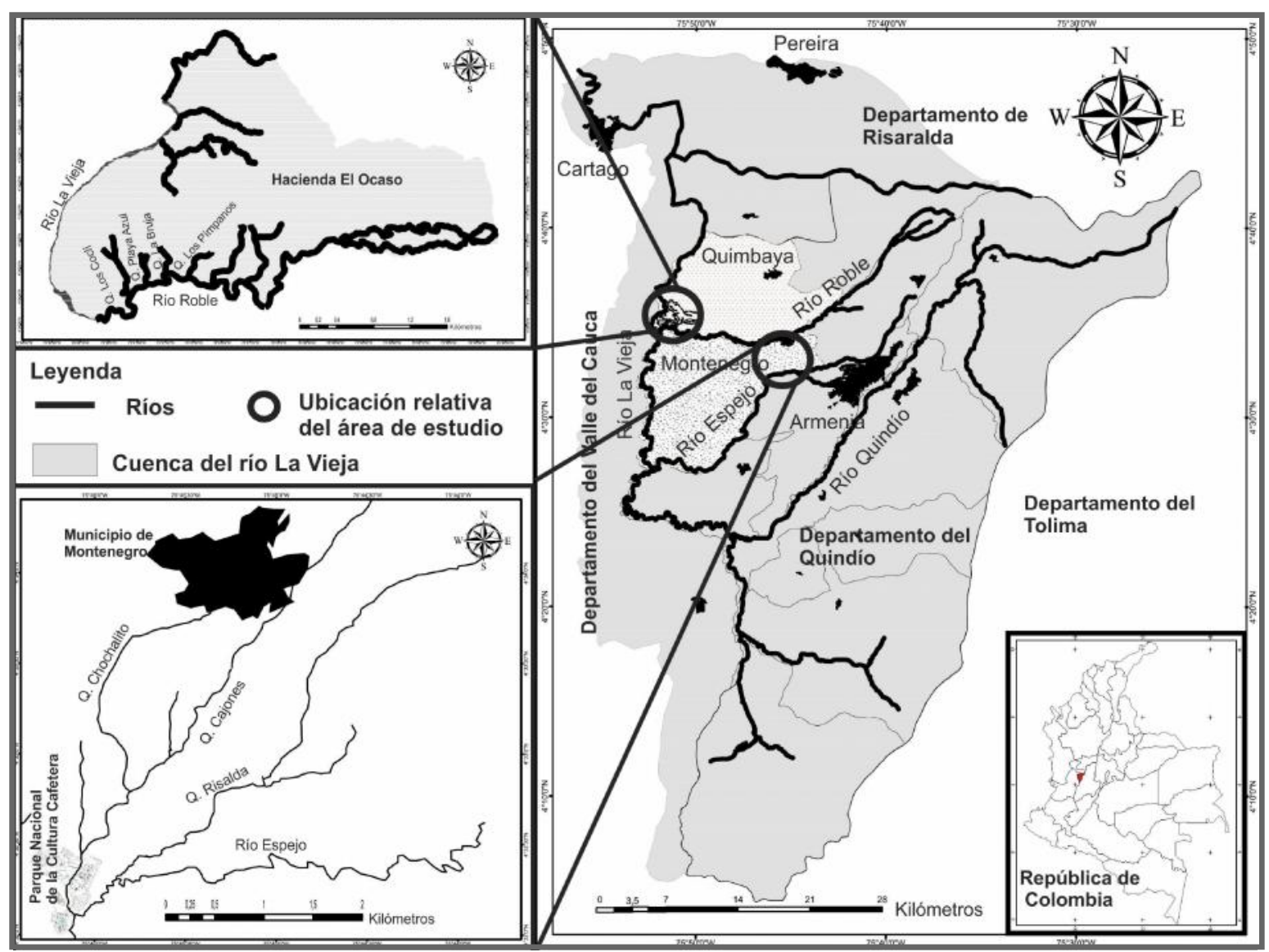

Figura 1. Mapa del departamento del Quindío con la ubicación de la quebrada Cajones y Los Coclí.

es relativamente más larga en los machos que en las hembras del mismo tamaño (Medem 1977). Además se observó el engrosamiento de la porción preanal, la cual es más gruesa en los machos por alojarse en esta región el hemipene (Corredor 2001).

Para establecer la proporción sexual en los capturados se tomó la talla del caparazón y peso de los individuos para inferir tallas cercanas a las etapas de madurez e inmadurez, debido a que aquellos individuos con longitud del caparazón igual o mayor de 20 $\mathrm{cm}$ pertenecían a individuos maduros sexualmente y aquellos con medida de longitud del caparazón menor de $20 \mathrm{~cm}$ pertenecían a individuos inmaduros sexualmente (protocolo de monitoreo 1994).

El marcaje de las tortugas se realizó con una muesca rectangular asignando valores a los escudos marginales y la suma de los valores con las muescas igualaban el número de ID del individuo. De esta manera se podía reconocer cuando una tortuga era recapturada y no repetir datos de medidas morfométricas (Rueda-Almonacid et al. 2007).
En las dimensiones corporales se tomaron los datos morfométricos de la longitud recta del caparazón (LCR), longitud curva del caparazón (LCC), anchura del caparazón (AC), altura del caparazón (AC), longitud recta del plastrón (LRP), longitud máxima del plastrón (LMC), longitud del puente (LP), longitud preanal (LPRC), longitud postanal (LPC) y el peso (Rueda-Almonacid et al. 2007).

La densidad de tortugas capturadas y avistadas se analizó mediante la fórmula $\mathrm{D}=\mathrm{ini} / \mathrm{i}$ ai, donde $n i$ fue el número de tortugas capturadas y avistadas en cada trayecto, ai fue el área de cada trayecto, calculando la densidad estimada de tortugas capturadas y avistadas por unidad de área (trayecto) (Greenwood y Robinson 2006). Seguido de la fórmula $\mathrm{D}=\mathrm{li} * \mathrm{Di} / \mathrm{L}$ para conocer la densidad estimada de tortugas por quebrada (Hurtado 1995). Para comparar la estructura de los individuos capturados y su distribución porcentual entre machos, hembras y juveniles se implementaron gráficos de barras. Se implementó la correlación de Pearson con el propósito de conocer 
el grado de asociación entre las variables morfométricas. Un análisis de regresión lineal, para conocer la relación entre las variables largo del caparazón y peso, largo del plastrón y peso, largo del plastrón y longitud preanal en machos y hembras (Montes y Ramírez 1978, Brower y Zar 1989, Krebs 1989, Styles 1998). Las correlaciones y regresiones solo fueron posibles con la población de la quebrada Cajones, pues los individuos capturados en la quebrada Los Coclí presentaron un $n=3$ lo que no permitió realizar estas interpretaciones.

\section{Resultados}

Densidad. En la quebrada Cajones se obtuvo registros de 38 tortugas de las cuales 20 fueron capturadas y 18 avistadas. De los capturados, 15 individuos adultos $(\mathrm{LC} \geq 20 \mathrm{~cm})$ divididos en siete machos y ocho hembras, además de cinco juveniles. En el trayecto uno se presentó un avistamiento y en el cuatro no se registraron individuos. Todas las capturas se efectuaron en los trayectos dos y tres. En el dos se encontraron seis hembras, tres machos y cuatro juveniles y en el tres, se capturaron dos hembras, cuatro machos y un juvenil. La mayoría de estos registros, se efectuaron en la mañana entre las 10-11 horas, en la tarde entre 13-14, 16-17 horas y en la noche entre las 19-22 horas.

La densidad registrada para la quebrada Cajones fue de 423,77 capturas y avistamientos $/ \mathrm{km}^{2}$. Para el trayecto número uno, se registró una densidad promedio 11,05 (valor mínimo 0 y máximo 714,29), presentado una alta variabilidad en los datos respecto al tamaño de la media y alta heterogeneidad en estos mismos $(\mathrm{CV}=2,45)$. Para el trayecto número dos, se registró una densidad promedio 757,69 (0-1876,17), presentado una baja variabilidad en los datos respecto al tamaño de la media y homogeneidad en los mismos en comparación con el anterior trayecto $(\mathrm{CV}=0,74)$. Para el número tres se registró la mayor densidad (promedio 818,33; mínimo 0-2659,57), presentado una baja variabilidad en los datos respecto al tamaño de la media y homogeneidad en los mismos $(\mathrm{CV}=0,75)$, al igual que en el trayecto número dos. Para el número cuatro se presentó la menor densidad con ausencia total de tortugas durante el estudio (Tabla 1).

En la quebrada Los Coclí, se obtuvo registros de cinco tortugas de las cuales tres fueron capturadas y dos avistadas. Entre los capturados se presentaron solo individuos juveniles (LC $\leq 20 \mathrm{~cm}$ ).

De acuerdo con la distribución espacial de las capturas, en el trayecto uno se presentó una captura; en el dos se capturaron dos individuos y se avistaron dos, todos los capturados y avistados fueron encontrados entre las 12-15 horas y de 18-19 horas. La densidad registrada para la quebrada Los Coclí fue de 275,34 capturas y avistamientos $/ \mathrm{km}^{2}$. Para el trayecto número uno se presentó una densidad promedio de 201,29 (dato mínimo 0; dato máximo 3623,19), con una alta variabilidad de los datos con respecto al tamaño de la media y alta heterogeneidad en el valor de los mismos $(\mathrm{CV}=4,25)$. El trayecto número dos presentó la mayor densidad de capturas y avistamientos $/ \mathrm{km}^{2}$ (promedio 349,41; mínimo 0-1572,33), con una menor variabilidad de los datos respecto al tamaño de la media y heterogeneidad en los mismos comparado con el anterior trayecto $(\mathrm{CV}=1,93)(\mathrm{Ta}-$ bla 2). La densidad poblacional fue diferente entre las quebradas (prueba de Kruskal-Wallis; p 0,00059) siendo mayor la densidad para la quebrada Cajones.

Estructura. En cuanto a la estructura de acuerdo con las tallas de los individuos adultos capturados en la quebrada Cajones, $50 \%$ presentó una talla entre $\mathrm{LC}=21,57-29,33 \mathrm{~cm}$ y $25 \%$ restante correspondió a tallas entre $\mathrm{LC}=32,33-39,13 \mathrm{~cm}$. El $25 \%$ correspondiente a juveniles presentó tallas de $\mathrm{LC}=13,93$ $18,93 \mathrm{~cm}$.

La mayor talla correspondió a una hembra con una longitud del caparazón de $\mathrm{LC}=39,13 \mathrm{~cm}$ y longitud del plastrón de $\mathrm{LP}=14,13 \mathrm{~cm}$, seguido de un macho con $L C=38,4 \mathrm{~cm}$ y $\mathrm{LP}=28,7 \mathrm{~cm}$; el ejemplar más pequeño capturado tuvo $\mathrm{LC}=13,93 \mathrm{~cm}$ y $\mathrm{LP}=10,13$ $\mathrm{cm}$.

En la distribución porcentual de los individuos de acuerdo con la longitud del plastrón, se registró que $65 \%$ presentó una talla entre $\mathrm{LP}=10-20 \mathrm{~cm}, 30 \%$ entre $\mathrm{LP}=20-30 \mathrm{~cm}$, siendo la mayor talla de $\mathrm{LP}=28,7$ $\mathrm{cm}$, perteneciente a un macho y $5 \%$ restante fueron tallas (iguales o menores a $10 \mathrm{~cm}$ ), con la menor $\mathrm{LP}=8,73 \mathrm{~cm}$ registrada para un macho (Figura 2).

Se encontró que los individuos capturados presentaron el caparazón más largo que el plastrón y que una vez alcanzaban la longitud del plastrón entre 20-30 cm este dejaba de crecer y el caparazón seguía aumentando de tamaño hasta una determinada 
Tabla 1. Densidad de tortugas en cada trayecto de muestreo de la quebrada Cajones.

\begin{tabular}{|c|c|c|}
\hline Trayectos quebrada Cajones & \multicolumn{2}{|c|}{ Promedio densidad (tortugas capturadas y avistadas en cada evento $/ \mathrm{km}^{2}$ ) } \\
\hline Trayecto \# 1 & $\begin{array}{l}\text { Promedio } \\
\text { Varianza } \\
\text { DE } \\
C V \\
\text { Máximo } \\
\text { Mínimo } \\
\text { Número de tortugas capturadas o avistadas }\end{array}$ & $\begin{array}{r}119,05 \\
85034,01 \\
291,61 \\
2,45 \\
714,29 \\
0 \\
1\end{array}$ \\
\hline Trayecto \# 2 & $\begin{array}{l}\text { Promedio } \\
\text { Varianza } \\
\text { DE } \\
\text { CV } \\
\text { Máximo } \\
\text { Mínimo } \\
\text { Número de tortugas capturadas o avistadas }\end{array}$ & $\begin{array}{r}757,69 \\
313079,03 \\
559,53 \\
0,74 \\
1876,17 \\
0 \\
21\end{array}$ \\
\hline Trayecto \# 3 & $\begin{array}{l}\text { Promedio } \\
\text { Varianza } \\
\text { DE } \\
\text { CV } \\
\text { Máximo } \\
\text { Mínimo } \\
\text { Número de tortugas capturadas o avistadas }\end{array}$ & $\begin{array}{r}818,33 \\
379738,41 \\
616,23 \\
0,75 \\
2659,57 \\
0 \\
16\end{array}$ \\
\hline $\begin{array}{l}\text { Trayecto \# } 4 \\
\text { Total de individuos capturados } \\
\text { o avistados } \\
\text { Densidad Quebrada } \text { km² }^{2}\end{array}$ & $\begin{array}{r}38 \\
423.77\end{array}$ & 0 \\
\hline
\end{tabular}

Tabla 2. Densidad de tortugas en cada trayecto de muestreo en la quebrada Los Coclí.

Promedio

Varianza

DE

$\mathrm{CV}$

Máximo

Mínimo

Número de tortugas capturadas o avistadas

Trayecto \# 2
Promedio

Varianza

DE

$\mathrm{CV}$

Máximo

Mínimo

Número de tortugas capturadas o avistadas
201,29

729305,23

853,99

4,24

3623,19

0

349,41

452431,01

672,63

1,93

1572,33 
Tabla 3. Diferencias morfométricas entre machos, hembras y juveniles de 20 individuos de C. acutirostris en la quebrada Cajones y 3 individuos en la quebrada Los Cocli.

\begin{tabular}{|c|c|c|c|c|c|c|c|c|c|}
\hline \multirow[t]{2}{*}{ Quebradas } & \multirow{2}{*}{$\begin{array}{l}\text { Variable/ } \\
\text { sexo }\end{array}$} & \multirow[t]{2}{*}{ Promedio } & \multirow[t]{2}{*}{ Varianza } & \multirow[t]{2}{*}{ DE } & \multirow[t]{2}{*}{ Rango } & \multirow[t]{2}{*}{$\mathbf{N}^{\circ}$} & \multicolumn{2}{|c|}{ Valor } & \multirow{2}{*}{$\begin{array}{l}\text { Coeficiente de } \\
\text { variación }\end{array}$} \\
\hline & & & & & & & máx. & $\min$. & \\
\hline \multicolumn{10}{|l|}{ Cajones } \\
\hline \multirow[t]{5}{*}{ Machos } & LC (cm) & 27,12 & 39,95 & 6,32 & $27,12 \pm 6,32$ & 7 & 38,04 & 21,57 & 0,23 \\
\hline & LP $(\mathrm{cm})$ & 19,08 & 41,71 & 6,45 & $19,08 \pm 6,45$ & 7 & 28,7 & 8,73 & 0,33 \\
\hline & LPre $(\mathrm{cm})$ & 8,93 & 3,65 & 1,91 & $8,93 \pm 1,91$ & 7 & 11,4 & 6,33 & 0,21 \\
\hline & LPost $(\mathrm{cm})$ & 18,77 & 7,38 & 2,71 & $18,77 \pm 2,71$ & 7 & 22,73 & 15,57 & 0,14 \\
\hline & P. (lb) & $\begin{array}{r}9,54 \\
N: 7\end{array}$ & 37,03 & 6,08 & $9,54 \pm 6,08$ & 7 & 22,58 & 4,97 & 0,63 \\
\hline \multirow[t]{6}{*}{ Hembras } & $\mathrm{LC}(\mathrm{cm})$ & 28,87 & 33,92 & 5,82 & $28,87 \pm 5,82$ & 8 & 39,13 & 22,93 & 0,2 \\
\hline & $\mathrm{LP}(\mathrm{cm})$ & 19,87 & 14,6 & 3,82 & $19,87 \pm 3,82$ & 8 & 24,73 & 14,13 & 0,19 \\
\hline & LPre. (cm) & 7,78 & 6,93 & 2,63 & $7,78 \pm 2,62$ & 8 & 13,67 & 5,47 & 0,33 \\
\hline & LPost. (cm) & 18,71 & 7,81 & 2,79 & $18,71 \pm 2,79$ & 8 & 22,33 & 14,93 & 0,14 \\
\hline & P.(lb) & 8,97 & 25,61 & 5,06 & $8,97 \pm 5,06$ & 8 & 18,47 & 3,91 & 0,56 \\
\hline & & & & & & & & & \\
\hline \multirow[t]{5}{*}{ Juveniles } & LC $(\mathrm{cm})$ & 16,12 & 4,52 & 2,12 & $16,12 \pm 2,12$ & 5 & 18,93 & 13,93 & 0,13 \\
\hline & $\mathrm{LP}(\mathrm{cm})$ & 11,8 & 2,62 & 1,62 & $11,8 \pm 1,62$ & 5 & 13,47 & 10,13 & 0,13 \\
\hline & LPre. (cm) & 4,12 & 1,23 & 1,11 & $4,12 \pm 1,11$ & 5 & 5,93 & 3,07 & 0,26 \\
\hline & LPost. (cm) & 11,97 & 3,31 & 1,82 & $11,97 \pm 1,82$ & 5 & 13,73 & 9,87 & 0,15 \\
\hline & P.(lb) & $\begin{array}{r}1,7 \\
N: 5\end{array}$ & 0,74 & 0,86 & $1,7 \pm 0,86$ & 5 & 2,84 & 0,43 & 0,5 \\
\hline \multirow{5}{*}{$\begin{array}{l}\text { Los Coclí } \\
\text { Juveniles }\end{array}$} & & & & & & & & & \\
\hline & $\begin{array}{l}\text { LC }(\mathrm{cm}) \\
\text { LP }(\mathrm{cm})\end{array}$ & $\begin{array}{l}16,16 \\
11.91\end{array}$ & $\begin{array}{l}2,53 \\
1.78\end{array}$ & $\begin{array}{l}1,59 \\
1.33\end{array}$ & $\begin{array}{l}16,16 \pm 1,59 \\
11,91+1,33\end{array}$ & $\begin{array}{l}3 \\
3\end{array}$ & $\begin{array}{l}17,17 \\
1293\end{array}$ & $\begin{array}{r}14,33 \\
104\end{array}$ & $\begin{array}{l}0,09 \\
0,11\end{array}$ \\
\hline & $\begin{array}{l}\text { LP }(\mathrm{cm}) \\
\text { LPre. }(\mathrm{cm})\end{array}$ & $\begin{array}{r}11,91 \\
4,55\end{array}$ & $\begin{array}{l}1,18 \\
0,79\end{array}$ & $\begin{array}{l}1,33 \\
0,89\end{array}$ & $4,55 \pm 0,89$ & 3 & $\begin{array}{r}1,50 \\
5,47\end{array}$ & 3,7 & 0,19 \\
\hline & LPost. (cm) & 11,4 & 1,24 & 1,11 & $11,4 \pm 1,11$ & 3 & 12,4 & 10,2 & 0,09 \\
\hline & $P \cdot(\mathrm{lb})$ & 1,37 & 0,62 & 0,79 & $1,37 \pm 0,79$ & 3 & 1,28 & 0,63 & 0,57 \\
\hline
\end{tabular}

$\mathrm{LC}=$ Longitud del caparazón, $\mathrm{LP}=$ Longitud del plastrón, $\mathrm{L}$ pre= Longitud preanal, $\mathrm{L}$ post= Longitud postanal, $\mathrm{P}=$ Peso

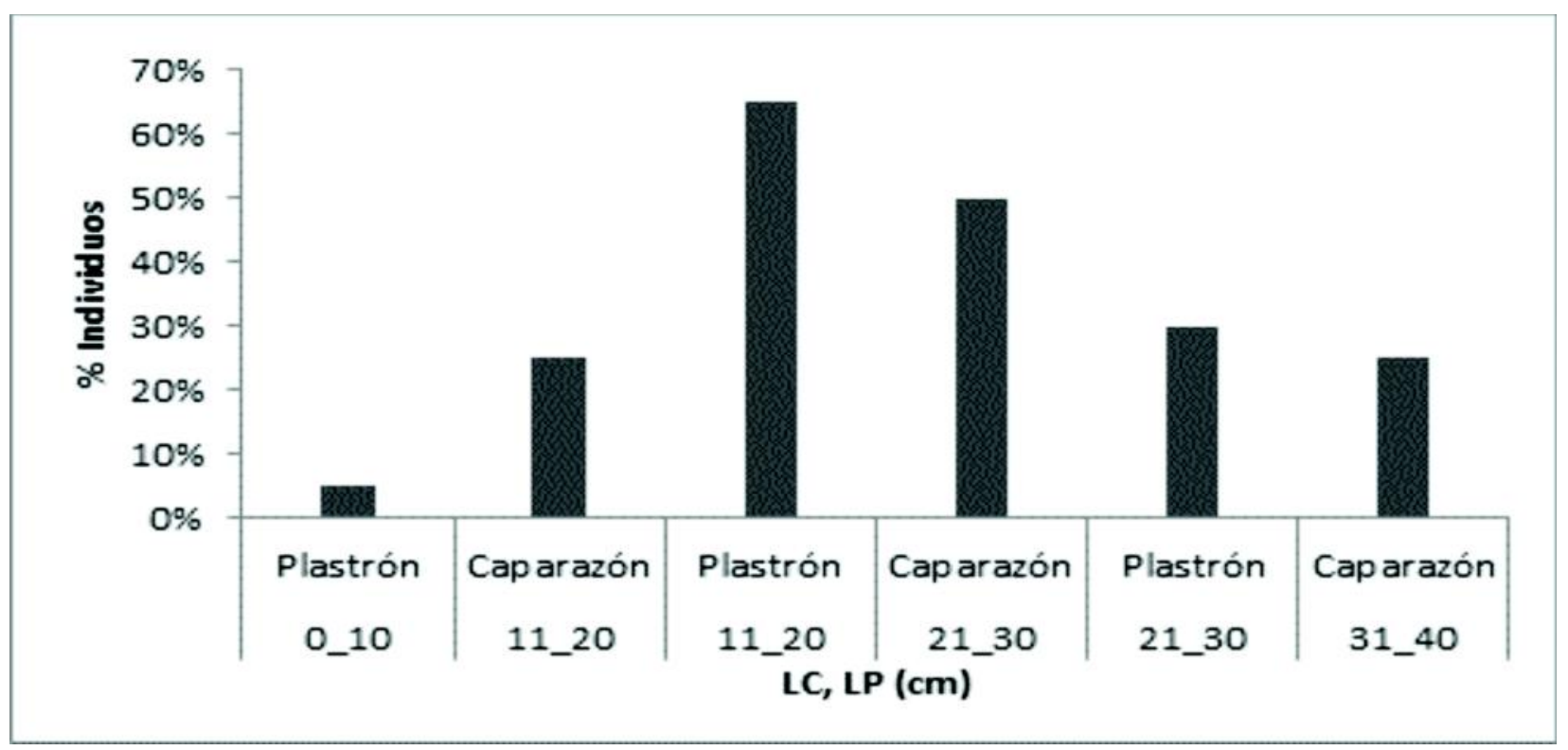

Figura 2. Distribución porcentual de 20 individuos de C. acutirostris de acuerdo con la longitud del caparazón (LC) y la longitud del plastrón (LP) en la quebrada Cajones. 
edad de la tortuga (Tabla 3). Las hembras fueron de mayor tamaño $(\mathrm{LC}=28,87 \mathrm{~cm})$ comparadas con los machos $(\mathrm{LC}=27,12 \mathrm{~cm})$, la longitud preanal $(7,78 \mathrm{~cm})$ y postanal $(18,71 \mathrm{~cm})$ de las hembras fue menor en relación con los machos (L. preanal $8,93 \mathrm{~cm}$, L. postanal $18,77 \mathrm{~cm})$, asimismo, el peso fue menor en las hembras $(8,97 \mathrm{~cm})$ a pesar de que tenían un mayor tamaño. En cuanto a los juveniles capturados se encontró que el tamaño una longitud del caparazón de 16,12 cm (valor máximo de 18,93, mínimo de 15,6) y un peso en promedio de 1,7 libras (valor máximo de 2,84 y un mínimo de 0,43 ) (Tabla 3 ).

En la quebrada Los Coclí el promedio de la LC de las tortugas fue de $16,16 \mathrm{~cm}$ y DE $1,59 \mathrm{~cm}$ en promedio (mínimo de 14,33; valor máximo $17,17 \mathrm{CV}$ 0,09), LP con un promedio de 11,91 ( $\mathrm{DE}=1,33$; CV 0.11 ), L. preanal con un promedio de 4,55 (DE $=0,89$ y $\mathrm{CV}=0,19)$. La longitud postanal con promedio de $11,4 \mathrm{~cm},(\mathrm{DE}=1,11 ; \mathrm{CV}=0,09)$, peso promedio de 1,37 lb. $(\mathrm{DE}=0,79 ; \mathrm{CV}=0,57)$ (Tabla 3$)$.

$\mathrm{Al}$ realizar la regresión lineal entre las variables largo del caparazón y peso en individuos adultos y juveniles de $C$. acutirostris, se encontró que los individuos de tallas menores, están altamente relacio$\operatorname{nados}(n=20, r=0,81, p=0,000013)$. Pero encontramos puntos atípicos que muestran que algunos individuos adultos llegan a un punto en que muy probablemente bajan la tasa de crecimiento de su caparazón y siguen aumentando de peso como lo es el caso de la hembra capturada con mayor longitud del caparazón de 39,13 cm y peso 44 libras (Figura 3).

El análisis entre el largo del plastrón y el peso en los individuos evaluados, no mostró relación alguna $(n=20, r=0,31, p=0,19)$, lo cual puede indicar que el aumento en la longitud del plastrón no está determinando el aumento del peso, ni viceversa.

La regresión lineal entre las variables corporales longitud del plastrón y longitud preanal en ocho hembras capturadas de $C$. acutirostris en la quebrada Cajones mostró una alta y positiva relación ( $\mathrm{n}=8 \mathrm{r}=0,70$ $\mathrm{p}=0,05)$ (Figura 3).

La regresión lineal entre las variables corporales longitud del plastrón y longitud preanal en siete machos de $C$. acutirostris en la quebrada Cajones mostró una alta y positiva relación $(\mathrm{n}=7 \mathrm{r}=0,75 \mathrm{p}=0,05)$ lo que indica que hubo un aumento de la longitud preanal con la longitud del plastrón en mayor proporción para los machos, logrando explicar que estos tienen la longitud preanal mucho más larga que las hembras (Figura 3).

La proporción sexual hallada en la quebrada Cajones fue de 1:1,1, lo que indica que hay una hembra por cada macho, permitiendo que esta población se encuentre en equilibrio.

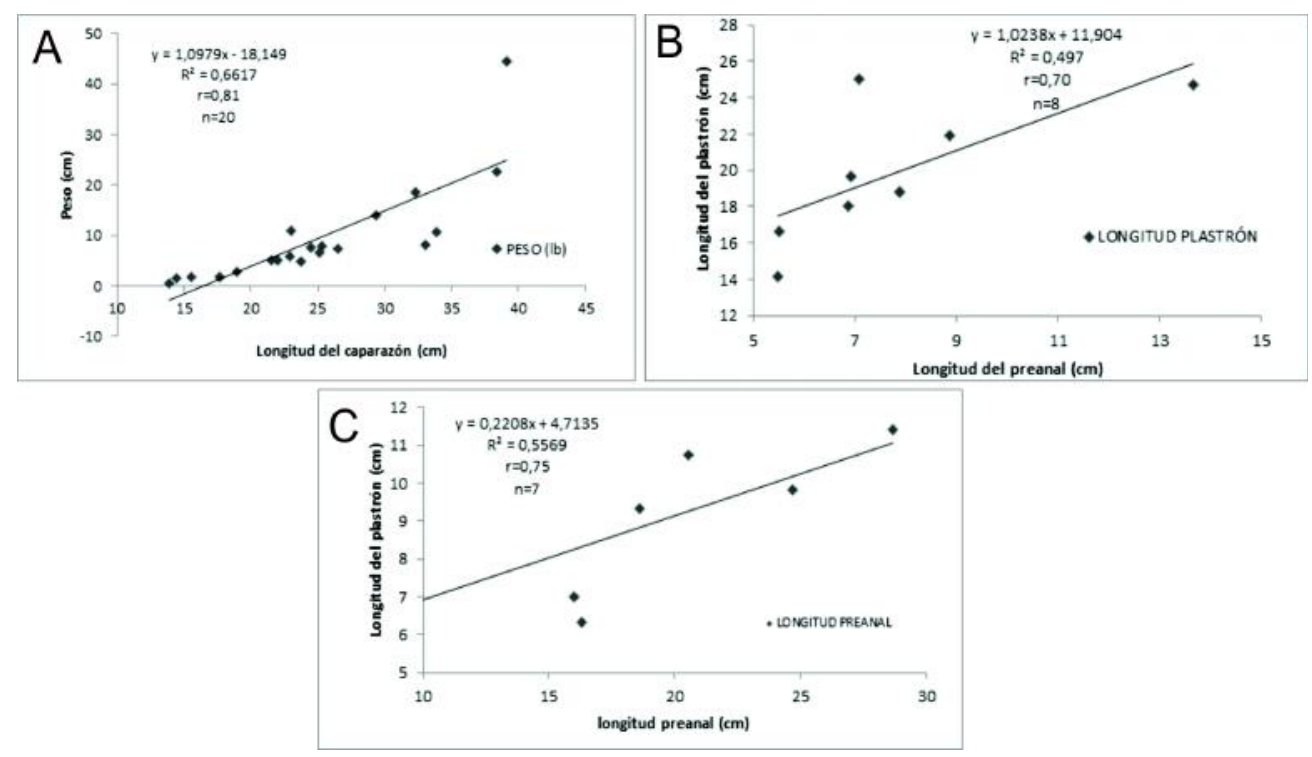

Figura 3. Regresiones lineales de variables morfométricas de C. acutirostris: A) entre las variables corporales: Largo caparazón (LC) y peso en individuos adultos y juveniles. B) Longitud preanal y longitud del plastrón en 8 hembras. C) Longitud preanal y longitud del plastrón en 7 machos. Todos los datos colectados son de individuos de la quebrada Cajones. 
Se registró para la quebrada Cajones la presencia de las siguientes especies de peces: Brycon sp., Hypostomus sp., Thrycomicterus sp., Cetopsorhamdia boquillae, Poecilia caucana, Trychomycterus striatus. Además, es de anotar que en la quebrada Los Coclí el día 14 de octubre se capturó una hembra ovipositando.

\section{Discusión}

La abundancia de una especie puede estar determinada por las condiciones microclimáticas, el alimento disponible y otros rasgos elusivos para el observador (Ceballos y Miranda 1986). La baja densidad en el trayecto número uno de la quebrada Cajones se puede deber a que este trayecto presenta aguas más claras comparado con los otros tres y baja disponibilidad de presas. Múnera y Regalado (2009) describen que las aguas claras y la poca disponibilidad de presas puede ser un factor limitante en la presencia de la tortuga; además, este sitio presenta un canal estrecho y baja profundidad; aquí se encuentra un puente que funciona como vía de acceso vehicular, zonas destinadas a cultivos y fincas cercanas que pueden generar disturbios a las tortugas y la alteración de su hábitat. Moll y Moll (2004) comentan que el destino de tierras para la agricultura y las construcciones pueden implicar cambios en el ambiente generando la reducción de las poblaciones de tortugas. También unos metros antes del final de este tramo se crea una barrera formada por rocas grandes y lisas que incrementan la velocidad de la corriente e impiden el desplazamiento de las tortugas hacia dicha área. Mosquera y Murillo (2003), Múnera y Regalado (2009) afirman que la velocidad de la corriente es uno de los factores que determina la presencia de la especie, razones que pudieron haber influido a encontrar una baja densidad para este sitio.

Los trayectos dos y tres fueron los que presentaron las mayores densidades para la quebrada Cajones; se encuentran ubicados en la parte media de la quebrada, son lugares con baja velocidad del agua, con los mayores valores de porcentaje de cobertura del dosel, profundidad del agua, profundidad del lodo, ancho del canal, turbidez del agua, zonas con una alta acumulación de nutrientes, alto contenido de materia orgánica en descomposición activa, troncos flotantes, cuevas bajo los barrancos y palizadas represadas de más de 100 metros de longitud que les brinda hábitat y refugio, variables similares a las encontradas por Medem (1977), Mosquera y Murillo (2003), Rueda-Almonacid et al. (2007), Múnera y Regalado (2009) y Páez et al. (2012) quienes reportaron que $C$. acutirostris prefieren zonas con estas características.

En estos dos sitios también se encontró una gran cantidad de vegetación acuática que sirve de alimento y camuflaje a las tortugas mientras acechan sus presas. Además hay playas de arena que podrían estar funcionando como sitios de nidación. Múnera y Regalado (2009) afirman que la disponibilidad de presas y la presencia de zonas para la nidación contribuyen positivamente en la presencia de la tortuga. Antúnez y Mendoza (1992) comentan que el microhábitat provee condiciones medio ambientales necesarias para llevar a cabo una variedad de funciones ecológicas. Debido a que solo estos dos sitios cumplen con los requerimientos biológicos y ecológicos de las tortugas pímpano, las lleva a concentrarse en estas zonas del cauce de la quebrada Cajones, razón por la cual se registró una mayor densidad para el trayecto dos $\mathrm{y}$ tres de esta quebrada. Si comparamos con el estudio de Mosquera y Murillo (2003) se encontró que los mayores registros de capturas se obtuvieron para las quebradas.

En el trayecto cuatro de la quebrada Cajones durante el estudio hubo ausencia total de individuos, esto se puede deber a que en este lugar son pocas las zonas lodosas, presentó baja temperatura el agua, producto de la alta cobertura de porcentaje del dosel, interviniendo en la incidencia de la energía solar para que la especie pudiera abastecer una variedad de necesidades, incluyendo la termorregulación, mediante la exposición directa de los rayos solares, en las playas, sobre troncos o exponiendo el dorso del caparazón sobre la superficie del agua y bajo la misma (Medem 1997, Rueda-Almonacid et al. 2007, Múnera y Regalado 2009). Esto les permite incrementar la temperatura corporal para acelerar los procesos digestivos, crecer y madurar los huevos, acondicionamiento de la piel y el caparazón y el retardo de infestaciones epizoóticas y epífitas (Mosquera y Murillo 2003, Boyer 1965, Rueda-Almonacid et al. 2007). Boyer (1965) y Reese y Welsh (1998) comentan que los sitios de asoleamiento son críticos en la termorregulación de las tortugas, particularmente 
cuando la temperatura del agua es baja, generando que las tortugas pasen más tiempo asoleándose, hasta que la temperatura del cuerpo de agua sea apta para permitir que los procesos fisiológico y energéticos sean adecuados para sus funciones como la alimentación, evitar ser depredados y la reproducción.

En la quebrada Los Coclí, la baja densidad de la tortuga en la parte alta puede estar relacionada con la presencia de pendientes pronunciadas en el cauce, así como en las riberas dificultando su desplazamiento a través de la quebrada, además de agua claras, sustratos rocosos, factores que no brindaban refugio a la especie frente a posibles depredadores. A medida que se desciende aumenta la turbidez en el agua así como la presencia de zonas fangosas y de inundación, disminuye el tamaño de sus rocas, factores que permiten el desplazamiento libre de las tortugas a través del cauce, vegetación que les brinda refugio y alimento, razón por la cual la densidad de tortugas aumentaba a medida que la quebrada cobraba identidad y porque las condiciones del terreno así lo permitían, además, el en trayecto dos sus zonas aledañas presentan lugares favorables para la anidación, ya que un mes después de haber llevado a cabo el muestreo en este mismo sitio se encontró una hembra grávida, es por ello que esta zona debe ser priorizada para la conservación.

Es probable que la razón por la que se encontraron individuos juveniles y la ausencia de adultos durante el muestreo en la quebrada Los Coclí, se deba a que las hembras visiten está quebrada en busca de lugares adecuados para dejar sus huevos y luego se marchen, una vez los huevos eclosionan salen los neonatos, alcanzan la talla juvenil y cuando completan su desarrollo y pasan a ser adultos abandonan este lugar en busca de mejores condiciones y recursos que puedan suplir sus requerimientos ecológicos y biológicos. Múnera y Regalado (2009) y Moll y Moll (2004) describen que las tortugas más grandes se pueden aventurar en áreas abiertas, mientras que las más pequeñas permanecen en zonas de protección conectadas con el cuerpo de agua, como en aguas estancadas, rara vez se aventuran en lo más profundo y en extensiones más amplias donde la velocidad de la corriente es mayor; además, la quebrada no cumple con los requerimientos para el establecimiento de individuos adultos, pues son cuerpos de agua pequeños, con bajo caudal y afectados drásticamente por las condiciones climáticas de bajas lluvias, generando que se sequen las zonas de inundación, disminuyendo y compactando el lodo que sirve de hábitat y refugio dejándolas al descubierto; los pozos que mantienen las pequeñas comunidades de peces y su conexión con la desembocadura del río, desaparecen temporalmente.

Un factor que puede estar afectando en gran medida la densidad de tortugas en el área de estudio es la cacería furtiva, pues el día 31 de octubre de 2012, se encontró una tortuga adulta desmembrada a orilla de la quebrada Cajones. Castaño-M (2002) reportan que estas tortugas son altamente consumidas y las condiciones económicas de los campesinos las convierten en un recurso alimenticio valioso. González (2005) reportó que la explotación comercial de huevos, crías y adultos como mascotas, aunado al deterioro y fragmentación de hábitat vitales para el normal desarrollo de las tortugas pímpano son las principales amenazas que aquejan la especie en el departamento del Quindío.

Otras de las amenazas registradas fue la deforestación y desecación de las zonas pantanosas, pues en la quebrada Cajones durante el estudio, se presentó la deforestación de una gran área al borde que generó la compactación del lodo en un sitio donde al inicio del estudio se encontraron tortugas juveniles enterradas y después de lo ocurrido no se encontraron más, por lo que se perdieron aquellas características del lugar que les permitía estar allí. RuedaAlmonacid (2007) afirma que las poblaciones de $C$. acutirostris localizada en el alto río Cauca han sido diezmadas como resultado de la sobre explotación y la desecación de estas áreas. Asimismo surge la problemática como destrucción o pérdida de hábitat y la pérdida de playas de anidación, ya sean como producto de la deforestación o por actividades como la extracción de balastro (Múnera y Regalado 2009, Moll y Moll 2004, Rueda-Almonacid et al. 2007).

El esfuerzo de muestreo empleado para la quebrada Cajones y la quebrada Los Coclí dependió del área en cada trayecto, pues aquellos trayectos con mayor área iban a tener un mayor esfuerzo de muestreo que aquellos de menor área. Al comparar el esfuerzo de muestreo empleado en el estudio de la tortuga pímpano en la quebrada Cristales por Mosquera y Murillo (2003), también dividieron los muestreos en un período de seis meses, con cuatro 
salidas por trayecto con duración de dos días y medio, además emplearon tres personas logrando registrar 28 individuos.

Al comparar el esfuerzo de muestreo que ellas emplearon fue mayor, razón por la que se obtuvo un mayor número de capturados. Múnera y Regalado (2009) para su estudio en los ríos Roble y Espejo, donde tres personas muestrearon durante diez meses, para un total de 43 trayectos, logrando obtener para el río Espejo 14 capturas y 20 avistamientos, mientras que para el río Roble solo se obtuvieron 3 avistamientos, a pesar de que no se encontró registro de la intensidad horas/trayecto, seguramente debió haber sido mayor el esfuerzo de muestreo comparado con este estudio; sin embargo, obtuvieron un menor número de capturas, esto se puede deber a que las mayores densidades de tortugas se están viendo concentradas en las quebradas, ya que estos sitios funcionan como zonas de refugio para la especie y pueden cumplir con sus requerimientos biológicos.

En la quebrada Cajones la población de tortugas presentó una alta proporción de adultos, el bajo número de juveniles puede estar relacionado con las altas tasas de mortalidad que se dan en estas etapas (Brooks et al. 1991). Mientras que para la quebrada Los Coclí solo se lograron capturar individuos juveniles, probablemente porque esta zona sea empleada para la anidación. La discontinuidad en los grupos de edades de tortuga pímpano en estas dos quebradas, junto con la presión antrópica sobre las mismas, podrían generar que las poblaciones sean altamente vulnerables a cualquier cambio en su densidad.

En general las tortugas capturadas en la quebrada Cajones fueron grandes, si se comparan con los datos en campo obtenidos por Múnera y Regalado (2009) quienes reportan $\mathrm{LC}=34,50 \mathrm{~cm}$, Mosquera y Murillo (2003) LC=36,2 cm, para la quebrada Cristales. En condiciones de cautiverio se ha logrado conseguir que las tortugas alcancen tallas superiores a $48 \mathrm{~cm}$ de LC (Zoológico de Cali). El individuo más pequeño capturado en la quebrada Cajones tenia dimensiones de $\mathrm{LC}=13,93 \mathrm{~cm}$, en la quebrada Los Coclí $\mathrm{LC}=14,33 \mathrm{~cm}$. Mosquera y Murillo (2003) registran un neonato con $\mathrm{LC}=3,7 \mathrm{~cm}$ y Múnera y Regalado (2009) un individuo de LC=3,4. Si comparamos los estudios realizados en el Quindío con C. acutirostris, se encuentra que las tortugas con mayor tamaño se tienen registradas en quebradas y los individuos más pequeños y juveniles se encuentran registrados para los ríos.

Es muy probable que los individuos adultos prefieran las quebradas porque estos generalmente permanecen largos períodos en un mismo lugar. Medem (1977) observó que los machos de esta especie pueden ocupar un territorio fijo, describiendo algunos comportamientos de territorialidad. Sin embargo, Ortega (2005) concluyó que no existía territorialidad ni jerarquía entre hembras y machos o entre juveniles y adultos. Además, estas tortugas una vez alcanzan la madurez sexual disminuye su tasa de depredación, por lo que la tortuga puede llegar a vivir más tiempo y lograr alcanzar un mayor tamaño. Es probable que los juveniles se expongan menos debido a la alta tasa de depredadores que presentan en esta etapa (Brooks et al. 1991) y a sus hábitos son más crípticos. Además, Congdon y Gibbons (1990) y Congdon et al. (1994) reportan que las tasas de mortalidad son naturalmente altas para las categorías huevos, neonatos y juveniles, y relativamente bajas para los subadultos y adultos. Sin descartar la probabilidad de que las metodologías implementadas en los estudios para quebradas hayan tenido sesgos en la captura de juveniles, aunque fueran muy rigurosas, por ello se obtuvo menor éxito en sus capturas.

Encontramos que los individuos de mayor tamaño fueron hembras y la longitud preanal y la longitud del plastrón aumentó una proporción más rápido en los machos que en las hembras, logrando obtener una alta y positiva relación entre estas dos variables. Mosquera y Murillo (2003) también reportaron un incremento más rápido de estas dos variables en los machos, al igual que lo reportado por Moisan y Bider (1965), White y Murphy (1973) concluyendo que el único indicador externo seguro del sexo es la región preanal la cual es más larga en los machos que en las hembras del mismo tamaño. Medem (1977) afirma que el sector preanal contiene el hemipene en todos los testudines y que en todos los ejemplares de $C$. acutirostris han encontrado el mayor tamaño en los machos. Lo mismo confirman Múnera y Regalado (2009) y Rueda-Almonacid et al. (2007) quienes reportan que los machos tienden a poseer la apertura de la cloaca en una posición más distal, el plastrón más pequeño y el puente más angosto. Pero difieren del presente estudio en que los individuos capturados de mayor tamaño fueron los machos y no las hembras. 
Es probable que en la zona de estudio las hembras estén presentando una mayor tasa de crecimiento, para alcanzar la madurez sexual antes que los machos, permitiendo que la energía requerida para generar un huevo esté disponible más temprano, lo que garantizaría una oferta de huevos constante en la población, además de considerar que estas tortugas presentan nidadas grandes lo que podría relacionarse con su tamaño corporal. Por otra parte, los machos podrían estar direccionando la energía que no invierten en su crecimiento, a suplir otro tipo de actividades como el incremento en la búsqueda de hembras o cortejo (Chen y Lue 2001 en Cuora flavomarginata) generando que se expongan con más frecuencia, lo que contribuye a ser depredados más fácilmente o sean extraídos por acción antrópica, disminuyendo la probabilidad de alcanzar mayores tallas que las hembras.

Cuando comparamos la longitud del caparazón (cm) con el peso (lb) en los 20 individuos de $C$. acutirostris capturados en la quebrada Cajones encontramos que fue estadísticamente significativa la regresión lineal, demostrando que las tortugas a medida en que aumentan la longitud del caparazón también ganan masa corporal y aquellos puntos que no se encontraron dentro de la línea de tendencia fueron aquellas tortugas que al alcanzar la etapa de madures disminuían su tasa de crecimiento en la longitud del caparazón. Esto se puede deber a que las tasas de crecimiento de los juveniles son mayores a la de los adultos, además cuando se inicia la reproducción las tasas de crecimiento corporal en individuos adultos son mucho más bajas que las tasas de crecimiento de los individuos inmaduros (Congdon y Gibbons 1990). Además, se debe tener en cuenta que se encontraron hembras con presencia de huevos lo que muy probablemente haya generado el aumento de sus pesos, debido a que esta especie se caracteriza por tener nidadas grandes (Páez et al. 2012).

Una vez se comparó la longitud del plastrón con el peso de los 20 individuos capturados en la quebrada Cajones, no encontramos una correlación significativa entre estas dos variables, lo cual indica que el aumento en la longitud del plastrón no determina el aumento de peso, lo contrario encontrado por Mosquera y Murillo (2003) quienes tienen una correlación positiva para la longitud del plastrón y el peso. $\mathrm{Al}$ efectuar la regresión lineal tampoco se encontró que estas dos variables estuvieran estadísticamente relacionadas. Esto también se puede deber al tamaño de la muestra que se manejó para cada estudio.

La población en la quebrada Cajones se encontró en equilibrio en términos de la proporción de machos y hembras 1:1, coincidiendo con lo reportado por Mosquera y Murillo (2003) para la quebrada Cristales y Múnera y Regalado (2009) para el río Espejo, donde la proporción sexual no fue significativamente diferente. El haber encontrado casi el mismo número de hembras que machos se puede deber en primer lugar a la temperatura de incubación, debido a que en la especie $C$. serpentina, la temperatura es la que determina el sexo en los neonatos (Yntema, 1979 en Chelydra serpentina citado por Páez et al. 2012) y tiene un patrón tipo II (TSD-II) que se relaciona con las hembras con temperaturas de incubación bajas y altas, y machos asociados con temperaturas de incubación intermedias (Páez et al. 2012, Ewert y Nelson 1991, Giraldo et al. 2012) de esta manera es muy probable que la presencia de machos y hembras en la quebrada Cajones esté asociada con que los nidos hayan estado expuestos a condiciones variables de temperatura que hayan tenido efecto sobre el sexo de los neonatos y permitan obtener una proporción equilibrada entre machos y hembras. Esta hipótesis debe ser comprobada experimentalmente.

En segundo lugar se tiene el acceso restringido a los lugares de estudio por ser áreas administradas en el caso de la quebrada Los Coclí por la Universidad del Quindío y la quebrada Cajones por el Parque Nacional de la Cultura Cafetera, han reducido el impacto por consumo humano y evitan transformaciones considerables en el hábitat; además, los únicos depredadores detectados para C. acutirostris durante esta investigación no tienen la capacidad de alimentarse de individuos adultos, solo de sus huevos y algunos de sus crías. Estos fueron el armadillo (Dasypus novemcinctus), guatín (Dasyprocta puntacta), coquito (Phimosus infuscatus), gallinazo (Coragyps atratus) y serpientes como Oxyrhopus petola, Chironius montícola, además, una vez el individuo supera el tamaño crítico, aumenta su probabilidad de llegar a la etapa adulta.

En la quebrada Los Coclí no se logró determinar la proporción de sexos debido a que solo se capturaron tres individuos y eran juveniles.

Durante el estudio para el mes de abril se captu- 
raron en la quebrada Cajones dos hembras con presencia de huevos y durante un monitoreo en la quebrada Los Coclí el día 14 de octubre se capturó una hembra ovipositando, el huevo era circular y tenía dimensiones de (longitud $=3,49 \mathrm{~cm}$, diámetro $=2,82 \mathrm{~cm}$, peso $=6,30 \mathrm{~g}$ ) medidas más pequeñas que las reportadas por Medem (1977) y Rueda-Almonacid et al. 2007). Medem (1977), informó una hembra anidante en el bajo Atrato en el mes de febrero, con huevos de forma circular y otros elipsoides, con dimensiones que variaban entre 3,5-3,3 cm (longitud y diámetro) y 3,9$3,2 \mathrm{~cm}$. Corredor et al. (2001) reportan un período de incubación de 110 días (rango 94-106 días) en hembras de $C$. acutirostris en el Zoológico de Cali, Colombia; Rueda-Almonacid et al. (2007) reportan que las posturas de $C$. acutirostris ocurren a comienzos del año, durante el mes de enero y los huevos son esféricos con diámetro de $37 \mathrm{~mm}$ y un peso de 5-15 g y comentan que el período de incubación tarda alrededor de tres meses. Mosquera y Murillo (2003) registraron neonatos en el mes de septiembre y esto las llevó a considerar que la época de anidación en la zona de estudio (quebrada Cristales) era en el mes de junio, coincidiendo con la época de bajas lluvias en el Quindío. Múnera y Regalado (2009) reportaron dos neonatos encontrados en la zona media del río Espejo para los meses de agosto y octubre, lo que coincidió con la temporada de cópula y nidación reportada por Meden (1977) y Rueda-Almonacid et al. (2007). Por consiguiente, la hembra de C. acutirostris que encontramos ovipositando en la quebrada Los Coclí, generó un nuevo reporte acerca de la época de anidación de la especie para la zona en el departamento del Quindío.

\section{Conclusiones}

Se encontraron diferencias entre la densidad poblacional de tortugas capturadas y avistadas de la quebrada Cajones y la quebrada Los Coclí, presentándose una mayor densidad para la quebrada Cajones. Por otro lado, la proporción de edades en las tortugas pímpano registradas para las quebradas Cajones y Los Coclí, indica que son vulnerables a cualquier factor que influya en la disminución de su densidad. A su vez, en la quebrada Cajones se encontró una proporción sexual en equilibrio, presentándose una hembra por cada macho. Igualmente en este sitio, las hembras presentaron mayores tamaños que los machos.

Se encontró una alta relación en las variables longitud del caparazón y peso en individuos juveniles y a su vez no se encontró relación entre las variables longitud del plastrón y peso en las tortugas capturadas. Por otro lado, existen diferencias significativas entre la distancia de la longitud preanal y longitud del plastrón en los machos con respecto a las hembras, siendo mayor para los machos.

\section{Agradecimientos}

Al Programa de Biología, Almacén de Laboratorio de Biología, Laboratorio de Aguas, GEHUQ (Grupo de Estudio en Herpetología de la Universidad del Quindío), por facilitar los equipos necesarios. Al Grupo de Investigación y Asesoría en Estadística, Universidad del Quindío, por el acompañamiento en el procesamiento de los datos, Parque Natural de la Cultura Cafetera por facilitar las instalaciones para la realización de este trabajo y a los auxiliares de campo Jhonatan W. Ocampo, Edilberto Quintero, Jhon Kenneddy Paniagua, Laura I. Roso Jaramillo, Luisa F. Franco, Luisa F. Sáenz, Santiago Styles, Ángela M. Morales, Jhonatan Culma y Jhon Deiber.

\section{Literatura citada}

Brower J, Zar C. 1989. Field and laboratory methods for general ecology. $3^{\text {a }}$ ed. Dubuque: Wm. C. Brown Publishers. p. 194.

Congdon JD, Gibbons JW. 1990. The evolution of turtle life histories. En: Gibbons JW (ed.). Life history and ecology of the slider turtle. Washington, DC, London: Smithsonian Institution Press. p.45-54.

Congdon JD, Dunham AE, Van Loben-S RC. 1994. Demographics of common snapping turtles (Chelydra serpentina): implications for conservation and management of longlived organisms. Am Zool. 34: 397-408.

Corredor GA, Espinoza DA, Galvis CA. 2001. Crianza en cautiverio de la tortuga bache (Chelydra acutirostris) en el zoológico de Cali, Colombia. Libro de resúmenes. V Congreso Internacional de Manejo de Fauna Silvestre en Amazonía y Latinoamérica.

Ewert MA, Nelson CE. 1991. Sex determination in turtles: diverse patterns and some possible adaptive values. Copeia. 1: 50-69.

Giraldo A, Garcés-Restrepo MF, Carr JL, Loaiza J. 2012. Tamaño y estructura poblacional de la tortuga sabaletera (Rhinoclemmys nasuta, testudines: Geoemydidae) en un ambiente insular del Pacífico colombiano. Caldasia. 34 
(1): 116-21.

Moll D, Edward OM. 2004. The ecology, explotation and conservation of river turtles. En: St. Cricq. Traditional explotation methods efficiency and consequences for river turtles. New York: Oxford University Press Inc. p. 83-6, 151-66.

Medem F. 1976. Recomendaciones respecto a contar el escamado y tomar las dimensiones de nidos, huevos y ejemplares de los Crocodylia y Testudines. Rev Lozania. 20: 1-17.

Medem F. 1977. Contribución al conocimiento sobre la taxonomía, distribución geográfica y ecológica de la tortuga «Bache» (Chelydra acutirostris). Caldasia. 12: 41-101.

Mosiman JE, Bider JR. 1960. Variation, sexual dimorphism and maturity in a Quebec population of the common snapping turtle, Chelydra serpentine. Can J Zool. 38: 19-38.

Mosquera S, Murillo S. 2003. Estado actual de la población de la tortuga pímpano Chelydra serpentina acutirostris en la quebrada Cristales del departamento del Quindio. (Tesis de pregrado). Armenia: Facultad de Educación, Universidad del Quindío. p. 54-78.

Múnera C, Regalado AC. 2009. Distribución y abundancia relativa actual de la tortuga pímpano (Chelydra acutirostris Peter 1862) Phillips et al.1996 en los ríos Roble y Espejo, sistema rio La Vieja, departamento del Quindío, Colombia. Universidad del Quindio. (Tesis de pregrado). Armenia: Facultad de Educación, Universidad del Quindío. p. 11-40.

Páez VP, Morales MA, Lasso CA, Castaño O, Bock BC. 2012.
Biología y conservación de las tortugas continentales de Colombia. Serie recursos hidrobiológicos y pesqueros continentales de Colombia. Bogotá: Instituto de Investigación de los Recursos Biológicos Alexander Von Humboldt (IAvH). p. 275-80.

Plan Básico de Ordenamiento Territorial. Municipio de Montenegro. Secretaria de Planeación Municipal. 2006. p.18-24.

Protocolo de monitoreo. 1994. Comisión Nacional de Áreas Naturales Protegidas. En: Monitoreo de la tortuga de cuatrociénegas (Terrapene coahuila) en el área de protección de flora y fauna cuatro ciénegas en el estado de Coahuila. México. Comisión Nacional de Áreas Naturales Protegidas. 8: 2-8.

Rueda-Almonacid JV. 2001. Programa nacional para la conservación de las tortugas marinas y continentales en Colombia. (Propuesta para concertar). Ministerio del Medio Ambiente. Dirección General de Ecosistemas. p. 17-32.

Rueda-Almonacid JV, Carr JL, Mittermeier RA, RodriguezMaecha JV, Mast RB, Vogt R. 2007. Las tortugas y los cocodrilianos de los países andinos del trópico. Serie de guías tropicales de campo $\mathrm{N}^{\circ} 6$. Conservación Internacional. Bogotá: Editorial Panamericana, formas e impresos. p. 90-251.

White JB, Murphy GG. 1973. The reproductive cycle and sexual dimorphism of the common snapping turtle, Chelydra serpentina serpentina. Herpetological. 29: 240-6. 